\title{
Incidence de Fusarium spp. associé aux semences de riz (Oryza sativa L.) au Burkina Faso
}

\author{
Fabrice Wendyam NIKIEMA ${ }^{1 *}$, Elisabeth Pawindé ZIDA ${ }^{1}$, Gilles I THIO ${ }^{1}$, \\ Léon W. NITIÉMA ${ }^{1}$, Kadidia KOITA ${ }^{2}$ et Mahamadou SAWADOGO² \\ IInstitut de l'Environnement et de Recherches Agricoles (INERA), Département Productions Végétales, 04 BP \\ 8645 Ouagadougou 04, Burkina Faso. \\ ${ }^{2}$ Université Joseph Ki-Zerbo. Département Biologie et Physiologie Végétales, Laboratoire Biosciences 03 BP \\ 7021, Ouagadougou 03, Burkina Faso. \\ *Auteur correspondant ; E-mail : wfnikiema@gmail.com; Tel : +226 76339922
}

\section{RESUME}

La production du riz au Burkina Faso est limitée par certaines maladies fongiques transmises par les espèces du genre Fusarium. A cet effet, l'importance de ces champignons a été évaluée sur 59 échantillons provenant de 7 variétés de riz par la méthode du papier buvard. Les sites sont répartis dans les 13 régions administratives situées en fonction des 3 zones agroécologiques du pays. Fusarium spp. a été identifié dans $81,35 \%$ des échantillons à des taux d'infection de 1 à $41 \%$. Fusarium spp. est présent dans toutes les zones agroécologiques avec des taux de prévalence variant de 78,2 à 83,87\%. Les échantillons provenant de la zone Soudano-Sahélienne et Soudanienne ont été plus infectés (respectivement 6,74\% et 5,34\%) que ceux de la zone Sahélienne $(1,40 \%)$. Ces champignons ont été rencontrés dans toutes les régions du pays avec des taux d'infections de semences variant de 0,5 à 15,25\%. Les régions les plus attaquées étant le Centre-Sud (15,25\%), le Centre (11,75\%) et la Boucle du Mouhoun (11,5\%). L'étude montre un taux d'infection global des semences par Fusarium spp. de 5,74\%. Une identification des espèces rencontrées sur le riz est impérative. L'évaluation de leur pathogénicité permettra de déterminer celles impliquées dans le gigantisme du riz.

(C) 2020 International Formulae Group. All rights reserved.

Mots clés : Fusarium spp., prévalence, taux d'infection, semences de riz

\section{Incidence of Fusarium spp. associated with rice seeds (Oryzae sativa $\mathbf{L}$.) in Burkina Faso}

\begin{abstract}
Rice production in Burkina Faso is limited by some fungal diseases transmitted by species from the genus Fusarium. To this end, the importance of these fungi was assessed on 59 samples from 7 rice varieties using the blotting paper method. The sites were distributed in the 13 administrative regions located according to the 3 agroecological zones of the country. Fusarium spp. was identified in $81.35 \%$ of the samples at infection rates ranging from 1 to $41 \%$. Fusarium spp. is present in all agroecological zones with prevalence rates ranging from 78.2 to $83.87 \%$. Samples from the Sudano-Sahelian and Sudanian zones were more infected $(6.74 \%$ and $5.34 \%$ respectively) than those from the Sahelian zone (1.40\%). These fungi were found in all regions of the country with seed infection rates ranging from 0.5 to $15.25 \%$. The most affected regions were the Centre-South (15.25\%),
\end{abstract}


the Centre $(11.75 \%)$ and the "Boucle du Mouhoun" (11.5\%). The study shows an overall seed infection rate of Fusarium spp. of 5.74\%. Identification of the species found on rice is imperative. Evaluation of their pathogenicity will make it possible to determine which species are involved in the rice's gigantism.

(C) 2020 International Formulae Group. All rights reserved.

Keywords: Fusarium spp., prevalence, infection rate, rice seed

\section{INTRODUCTION}

Le riz (Oryza sativa L.) est une culture vivrière importante et une source de revenus pour nombreux ménages agricoles en Afrique. La demande de riz en Afrique subsaharienne augmente plus rapidement que la production (Van oort et al., 2015). Le riz est devenu un produit très stratégique et prioritaire pour la sécurité alimentaire en Afrique. $\mathrm{La}$ consommation augmente plus rapidement que tout autre produit de base sur le continent du fait de la croissance démographique importante, de l'urbanisation rapide et de l'évolution des habitudes alimentaires (Seck et al., 2013).

Au Burkina Faso, le riz occupe la $4^{\text {ème }}$ place parmi les céréales cultivées, tant du point de vue des superficies que de la production. La production nationale de riz est estimée à 325566 tonnes pour une superficie de 165086 hectares en 2018 (DGESS, 2019). Le riz est cultivé dans la quasi-totalité des 13 régions administratives du pays, avec tout de même des régions à très forte production comme les Haut bassins, le Centre-Est, la Boucle du Mouhoun et les Cascades (CIR-B, 2011). Les variétés de riz améliorées les plus utilisées par les producteurs sont la FKR 19 (34,34\%), la FKR $14(11,11 \%)$ et la FKR $34(4,04 \%)$ (Ouédraogo et Dakouo, 2017). Les besoins en riz augmentent rapidement avec la croissance démographique, en particulier dans les zones urbaines (Badolo et Traoré, 2015). La production nationale ne couvre pas les besoins alimentaires du pays, et face à la forte demande des populations en riz, le pays importe cette denrée. En effet, le Burkina Faso importe chaque année environ 300000 tonnes de riz d'une valeur estimée à 40 milliards de francs CFA pour sa consommation locale (MASA, 2013). Pour accroître la production et réduire les importations, des stratégies nationales de développement de la riziculture ont été mises en place (Bila, 2015) et ont consisté notamment à l'aménagement et l'exploitation de 2500 ha de bas-fonds en 5 ans, l'organisation, l'encadrement et le suivi des producteurs par les techniciens de l'agriculture et les différentes structures habilités ainsi que la poursuite des subventions pour les intrants agricoles dont les fertilisants et les semences améliorées. Cela a entraîné un accroissement de la production qui est passée de 270658 tonnes en 2010 à 350392 tonnes en 2018. Malgré cet essor de la production dû principalement à l'augmentation des superficies aménagées au niveau des bas-fonds et des périmètres irrigués, la riziculture burkinabè reste confrontée à des défis majeurs liés aux effets de facteurs abiotiques (températures défavorables, inondations, sècheresse, etc.) et biotiques (maladies, insectes, oiseaux, mauvaises herbes). Parmi les maladies fongiques du riz, le gigantisme du riz (Bakanae), causé par des espèces de Fusarium dont Fusarium fujikuroi et $F$. concentricum, constitue une maladie émergente dans plusieurs pays producteurs de riz (Desjardins et al., 2000 ; Bashyal et al., 2016 ; Jeon et al., 2013 ; Raghu et al., 2018). Le Bakanae est responsable de pertes de rendement élevées allant de 3,0 à 95,4\% (Gupta et al., 2015). Le genre Fusarium est considéré comme l'un des plus importants groupes d'agents pathogènes des plantes, car il contient un nombre d'espèces qui sont distribuées dans le monde entier et s'attaquent à de nombreux secteurs agricoles et horticoles d'importance économique (Ma et al., 2013). L'analyse de la mycoflore des grains de différentes variétés de riz a mis en évidence des espèces fongiques très diversifiées, notamment celles du genre Fusarium, au Burkina Faso (Kini et al., 2002; Mathur et Manandhar, 2003 ; Ouédraogo et al., 2016). Cependant, au 
plan national, peu d'informations existent sur le gigantisme, son expansion géographique, les principales espèces fongiques impliquées dans l'infection. C'est pour contribuer à une meilleure connaissance de la maladie au Burkina Faso et envisager une stratégie de lutte adéquate de gestion que cette étude a été réalisée. Elle a pour objectif d'évaluer la prévalence, l'incidence et la distribution de Fusarium spp. associé aux semences de riz produites au Burkina Faso.

\section{MATERIEL ET METHODES}

\section{Collecte des échantillons de semences}

Les opérations de collecte d'échantillons de semences de riz ont été conduites en 2018 à travers tout le pays et ont concerné différentes localités situées dans les trois zones climatiques. Elles ont été réalisées dans 59 sites localisés dans 31 provinces (Figure 1). Ces provinces sont réparties dans les 13 régions administratives du pays en fonction des différentes zones climatiques (Sahélienne, Soudano-Sahélienne et Soudanienne) du Burkina Faso. Les caractéristiques des zones climatiques sont les suivantes: la zone Sahélienne avec une pluviométrie annuelle de $300-600 \mathrm{~mm}$, a une température moyenne annuelle de $29{ }^{\circ} \mathrm{C}$. Les zones Soudano-Sahélienne et Soudanienne présentant respectivement $600-900 \mathrm{~mm}$ et 900 $1200 \mathrm{~mm}$ de pluviométrie annuelle, affichent des températures annuelles respectives de 28 ${ }^{\circ} \mathrm{C}$ et $27^{\circ} \mathrm{C}$ (MECV., 2007).

Les échantillons de semences de sept (7) variétés de riz dont six variétés améliorées (FKR19, FKR45N, TS2, FKR56, FKR78, NERICA4) et une variété locale ont été collectés auprès des producteurs. La taille des échantillons a varié de 500 à $1000 \mathrm{~g}$. Chaque échantillon collecté a été accompagné d'une fiche informative complète comportant: le code d'identification de l'échantillon, le nom du site de production, les coordonnées GPS, la date de collecte, le nom de la variété, le nom du producteur, l'année de production de la semence. Ces échantillons sont constitués de semences produites pendant la saison agricole 2017-2018.

\section{Conservation des échantillons de semences au laboratoire}

Au laboratoire, un échantillon de travail constitué de 400 grains a été prélevé à partir de chaque échantillon de semences, pour l'étude et le reste des semences a été stocké dans un réfrigérateur et conservé $4{ }^{\circ} \mathrm{C}$.

\section{Analyse sanitaire des semences après incubation (méthode du papier buvard)}

La méthode standard du papier buvard a été utilisée pour détecter les champignons du genre Fusarium capables de se développer sur les semences en présence d'humidité. Quatre cent (400) semences pures de chaque échantillon ont été disposées dans des boîtes de Pétri $(90 \mathrm{~mm}$ de diamètre) contenant trois couches de papier buvard humidifié, à raison de 25 grains par boîte. Les boîtes ont été placées dans une chambre à $20-25^{\circ} \mathrm{C}$, sous des cycles d'éclairages alternatifs de 12 heures de lumière proche ultraviolette et 12 heures d'obscurité par jour, pendant sept jours. Chaque échantillon a été analysé suivant un dispositif blocs randomisés à 4 répétitions, soit 100 grains par répétition. Les grains incubés ont été examinés individuellement au stéréo microscope pour détecter la présence ou l'absence de champignons. L'identification a concerné uniquement les champignons appartenant au genre Fusarium et a été confirmée par un examen du mycélium et/ou des conidies au microscope et en se référant au manuel d'identification des champignons de Mathur et Kongsdal (2003). Les Fusarium présents sur chaque grain, quelle que soit l'espèce, ont été notés et le pourcentage de grains infectés par Fusarium spp. a été calculé pour chaque échantillon de semences selon la formule :

$\mathrm{Ti}(\%)=[($ Nombre de grains infectés par Fusarium) / N] X 100.

Où $\mathrm{Ti}=$ Taux d'infection des semences par Fusarium spp. ; $\mathrm{N}=$ Nombre total de grains examinés par échantillon de semences $(\mathrm{N}=400)$.

La prévalence $(\mathrm{P})$ ou pourcentage d'échantillons de semences contaminés par Fusarium, quelle que soit l'espèce, a également été calculé suivant la formule : 
$\mathrm{P}(\%)=[($ Nombre d'échantillons de semences contaminés par Fusarium spp.) / M] X 100 .

Où $\mathrm{P}=$ Proportion d'échantillons de semences contaminés par Fusarium spp. ; M = Nombre total d'échantillons de semences examinés.

\section{Isolement et conservation des isolats de Fusarium}

Fusarium spp. a été isolé à partir des semences de riz incubées sur lesquelles ces champignons se sont développés sous forme de mycélium. A cet effet, un fragment de mycélium des champignons étudiés a été prélevé et déposé sur le milieu de culture PDA (Patato Dextrose Agar) composé d'un litre d'eau distillée, $4 \mathrm{~g}$ d'infusât de pomme de terre, $20 \mathrm{~g}$ de $\mathrm{D}(+)$-glucose et $15 \mathrm{~g}$ d'agar. Des repiquages successifs ont permis la purification des différents champignons en culture et leur conservation à une température de $4{ }^{\circ} \mathrm{C}$.

\section{Analyses statistiques}

Le tableur Excel 2010 a été utilisé pour la saisie et l'organisation des données ainsi que la réalisation des graphiques. Les données sur la Prévalence et le Taux d'infection des semences de riz par Fusarium spp. ont été soumises à des analyses de variance (ANOVA) à l'aide du logiciel SAS. La séparation des moyennes a été faite par le test de Newmans Keuls au seuil de $5 \%$.

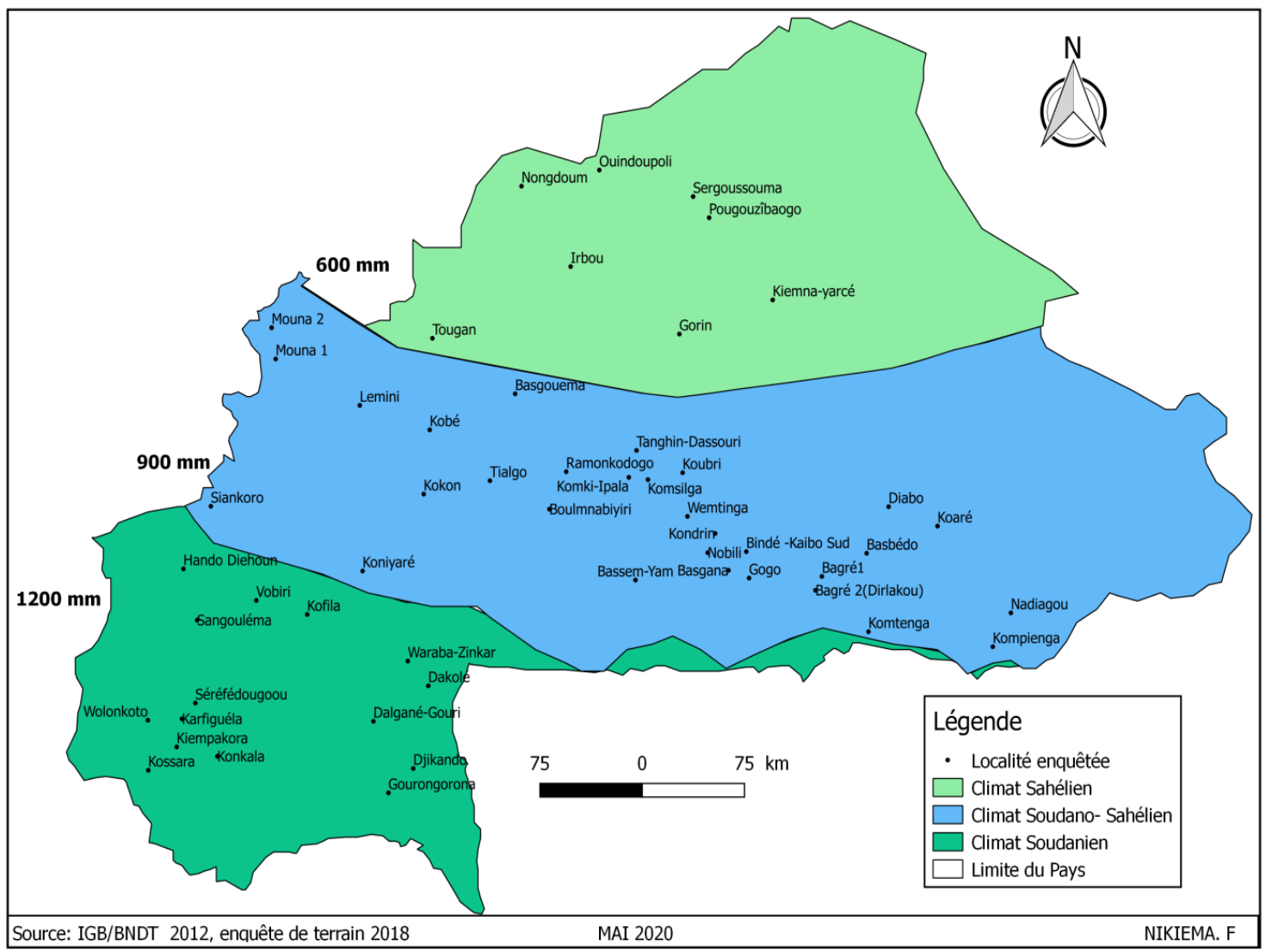

Figure 1 : Sites de collecte des échantillons de semences de riz au Burkina Faso en 2017-2018. 
RESULTATS

\section{Résultats de la collecte des échantillons de semences}

$\mathrm{Au}$ total, 59 échantillons de semences ont été collectés dans 59 localités réparties dans les trois zones agro-écologiques du pays. Parmi ces échantillons de semences, 36 échantillons (soit 61,02\%) proviennent de la zone soudanosahélienne, 18 échantillons (soit 30,51\%) proviennent de la zone soudanienne et 5 échantillons (soit 8,47\%) proviennent de la zone sahélienne. On note que la grande majorité des échantillons de semences de riz provient des zones soudano-sahélienne et soudanienne avec respectivement $61,02 \%$ et $30,51 \%$, tandis que dans la zone sahélienne, le nombre d'échantillons collectés a été relativement faible.

Prévalence de Fusarium spp. sur le riz et taux d'infection de semences produites au Burkina Faso

Les résultats portant sur les niveaux d'infection des semences des différents échantillons par Fusarium spp. ont été présentés dans le Tableau 1. Sur l'ensemble des 59 échantillons de semences collectés à travers le pays, Fusarium spp. a été détecté sur 48 échantillons, soit un taux de prévalence national de $81,35 \%$. Les taux d'infection des semences ont été variables suivant les échantillons et ont été compris entre 0 et $41 \%$ (Tableau 1). Parmi les échantillons de semences collectés, 11 ont été indemnes de Fusarium, 38 ont présenté des taux de contamination allant de 1 à 10\%, 06 échantillons ont montré des taux d'infection allant de 11 à $16 \%$ et 04 échantillons ont enregistré entre 21 et $41 \%$ de semences infectées par Fusarium spp.

\section{Prévalence et Taux d'infection par} Fusarium spp. de semences de riz en fonction des zones agroécologiques de production du riz

Sur les 48 échantillons de semences infectés par Fusarium spp., 32, 12 et 4 proviennent respectivement de la zone soudano-sahélienne, de la zone soudanienne et de la zone sahélienne, ce qui correspond à des taux de prévalence respectifs de $88,89 \%$, $66,67 \%$ et $80 \%$ observés dans lesdites zones (Tableau 2). Les champignons ont été rencontrés dans les semences dans toutes les zones agroécologiques à des taux moyens d'infection compris entre $1,40 \%$ et $7,72 \%$. Les semences produites dans la zone sahélienne, avec $1,4 \%$ de taux moyen d'infection, ont été statistiquement moins contaminées par Fusarium spp. que celles produites dans les zones soudano-sahélienne $(7,72 \%$ de taux moyens de contamination) et soudanienne $(3,00 \%)$.

Prévalence et Taux d'infection des semences de riz par Fusarium spp. en fonction des régions administratives du Burkina Faso.

Les résultats d'analyse sanitaire des différents échantillons de semences de riz ont montré la présence de Fusarium spp. dans toutes les régions administratives du pays. Les taux de prévalence ont varié de 25 à $100 \%$ (Figure 2). Les échantillons collectés dans les régions du Centre, Centre-Sud, Centre-Ouest, Centre-Nord, Plateau-Central et de la Boucle du Mouhoun ont été tous contaminés par Fusarium spp. pendant que dans la Région de l'Est, seulement $25 \%$ des échantillons de semences étaient contaminés. Les taux d'infection des semences dans les régions ont varié de 0,5 à 15,25\% (Figure 2). Les semences produites dans les régions du Centre-Sud, du Centre et de la Boucle du Mouhoun ont été significativement plus attaquées par Fusarium spp. avec en moyenne, respectivement $15,25 \%$, $11,75 \%$ et $11,52 \%$ de taux d'infection, que celles produites dans les autres régions $(0,5$ $6,67 \%$ de taux d'infection). Les semences produites dans la région de l'Est étant les plus faiblement contaminées $(0,5 \%$ de taux moyen d'infection).

Taux d'infection des semences par Fusarium spp. selon la variété d'appartenance des échantillons de semences

Les échantillons de semences collectés proviennent de sept variétés de riz dont six améliorées et une locale. Il s'agit de : FKR19 (35 échantillons de semences), FKR45N (3 
échantillons), TS32 (15 échantillons), FKR56 (3 échantillons), et, FKR78, NERICA4 et la variété locale représentée chacune par un seul échantillon (Tableau 3). Pour chaque variété, le nombre d'échantillons de semences contaminés par Fusarium spp. a été le suivant : 30 échantillons (soit 85,71\%) pour FK19; 13 échantillons $(86,67 \%)$ pour TS2; deux échantillons $(66,67 \%)$ pour FKR56; un échantillon $(33,33 \%)$ pour FKR45N ; un échantillon (100\%) pour FKR78 et NERICA et enfin zéro échantillon $(0 \%)$ pour la variété locale. La variété locale, avec l'unique échantillon collecté, a été exempte d'infection.

Les niveaux d'infection des semences des différentes variétés par Fusarium spp. ont varié de 0 à 6,90\% (Tableau 3). Les analyses statistiques n'ont indiqué aucune différence significative entre ces taux d'infections. La variété locale n'a pas été infectée par Fusarium spp. comparativement aux variétés améliorées.

Tableau 1 : Taux d'infection par Fusarium spp. de 59 échantillons de semences collectés dans différentes localités du Burkina Faso en 2018.

\begin{tabular}{|c|c|c|c|c|}
\hline Echantillons & $\begin{array}{l}\text { Zones Agro- } \\
\text { écologiques }\end{array}$ & $\begin{array}{l}\text { Régions } \\
\text { administratives }\end{array}$ & Localités & $\begin{array}{l}\text { Taux d'infection par } \\
\text { Fusarium spp. (\%) } \\
\end{array}$ \\
\hline $\mathrm{CS} 4$ & Soudano-Sahélienne & Centre-Sud & Gogo & $41,000 \mathrm{a}$ \\
\hline $\mathrm{C} 2$ & Soudano-Sahélienne & Centre & Komsilga & $30,000 \mathrm{~b}$ \\
\hline CS6 & Soudano-Sahélienne & Centre-Sud & Basgana & $24,000 \mathrm{c}$ \\
\hline BM1 & Soudano-Sahélienne & Boucle du Mouhoun & Gobé & $21,000 \mathrm{~cd}$ \\
\hline BM2 & Soudano-Sahélienne & Boucle du Mouhoun & Moundasso1 & $16,000 \mathrm{de}$ \\
\hline BM3 & Soudano-Sahélienne & Boucle du Mouhoun & Moundasso2 & $15,000 \mathrm{def}$ \\
\hline $\mathrm{C} 3$ & Soudano-Sahélienne & Centre & Koubri & 12,000 efg \\
\hline $\mathrm{Ca} 1$ & Soudanienne & Cascades & Séréfédougou & 12,000 efg \\
\hline $\mathrm{CS} 2$ & Soudano-Sahélienne & Centre-Sud & Kondrin & 11,000 efg \\
\hline BM6 & Soudano-Sahélienne & Boucle du Mouhoun & Tougan & 11,000 efg \\
\hline HB3 & Soudanienne & Haut-bassins & Kofila & 10,000 efg \\
\hline CE3 & Soudano-Sahélienne & Centre-Est & Bagré1 & 9,000 efg \\
\hline $\mathrm{N} 2$ & Soudano-Sahélienne & Nord & Basgouema & 8,000 efg \\
\hline $\mathrm{SO} 4$ & Soudanienne & Sud-Ouest & Djikando & 8,000 efg \\
\hline $\mathrm{PC} 1$ & Soudano-Sahélienne & Plateau-Central & Signoghin & 8,000 efg \\
\hline $\mathrm{N} 1$ & Soudano-Sahélienne & Nord & Irbou & 7,500 efg \\
\hline PC3 & Soudano-Sahélienne & Plateau-Central & Napamboumbou & 7,000 efg \\
\hline $\mathrm{CO} 6$ & Soudano-Sahélienne & Centre-Ouest & Boulmnabyiri & 7,000 efg \\
\hline $\mathrm{CS} 1$ & Soudano-Sahélienne & Centre-Sud & $\begin{array}{l}\text { Bindé-Kaibo } \\
\text { Sud }\end{array}$ & 7,000 efg \\
\hline $\mathrm{CS} 3$ & Soudano-Sahélienne & Centre-Sud & Wetenga & 6,500 efg \\
\hline $\mathrm{PC} 2$ & Soudano-Sahélienne & Plateau-Central & Kolgondiéssé & $5,000 \mathrm{fg}$ \\
\hline $\mathrm{SO} 2$ & Soudanienne & Sud-Ouest & Dalgané-Gouri & $5,000 \mathrm{fg}$ \\
\hline $\mathrm{CO} 5$ & Soudano-Sahélienne & Centre-Ouest & Ramonkodogo & $5,000 \mathrm{fg}$ \\
\hline BM4 & Soudano-Sahélienne & Boucle du Mouhoun & Lémini & $4,000 \mathrm{~g}$ \\
\hline $\mathrm{C} 4$ & Soudano-Sahélienne & Cenrre & Komki-Ipala & $4,000 \mathrm{~g}$ \\
\hline
\end{tabular}




\begin{tabular}{|c|c|c|c|c|}
\hline HB2 & Soudanienne & Haut-bassins & Koniyaré & $3,000 \mathrm{~g}$ \\
\hline $\mathrm{Ca} 3$ & Soudanienne & Cascades & Konkala & $3,000 \mathrm{~g}$ \\
\hline $\mathrm{SO} 3$ & Soudanienne & Sud-Ouest & Dakolé & $3,000 \mathrm{~g}$ \\
\hline $\mathrm{Ca} 5$ & Soudanienne & Cascades & Kiempakora & $3,000 \mathrm{~g}$ \\
\hline $\mathrm{CN} 2$ & Sahélienne & Centre-Nord & Garlaré & $3,000 \mathrm{~g}$ \\
\hline CS5 & Soudano-Sahélienne & Centre-Sud & Nobili & $2,000 \mathrm{~g}$ \\
\hline $\mathrm{E} 4$ & Soudano-Sahélienne & Est & Diabo & $2,000 \mathrm{~g}$ \\
\hline HB4 & Soudanienne & Haut-bassins & Vobiri & $2,000 \mathrm{~g}$ \\
\hline $\mathrm{Ca} 4$ & Soudanienne & Cascades & Karfiguela & $2,000 \mathrm{~g}$ \\
\hline HB1 & Soudanienne & Haut-bassins & Hando Diehoun & $2,000 \mathrm{~g}$ \\
\hline N4 & Soudano-Sahélienne & Nord & Masboré & $2,000 \mathrm{~g}$ \\
\hline $\mathrm{CO} 4$ & Soudano-Sahélienne & Centre-Ouest & Tialgo & $2,000 \mathrm{~g}$ \\
\hline $\mathrm{CO} 3$ & Soudano-Sahélienne & Centre-Ouest & Bassemyam & $2,000 \mathrm{~g}$ \\
\hline $\mathrm{S} 2$ & Sahélienne & Sahel & Sergoussouma & $2,000 \mathrm{~g}$ \\
\hline BM5 & Soudano-Sahélienne & Boucle du Mouhoun & Kokoun & $2,000 \mathrm{~g}$ \\
\hline CE4 & Soudano-Sahélienne & Centre-Est & Bagré2 & $2,000 \mathrm{~g}$ \\
\hline CE2 & Soudano-Sahélienne & Centre-Est & Bassedo & $2,000 \mathrm{~g}$ \\
\hline HB5 & Soudanienne & Haut-bassins & Siankoro & $1,000 \mathrm{~g}$ \\
\hline $\mathrm{C} 1$ & Soudano-Sahélienne & Centre & $\begin{array}{l}\text { Tanghin- } \\
\text { Dassouri }\end{array}$ & $1,000 \mathrm{~g}$ \\
\hline $\mathrm{CN} 1$ & Sahélienne & Centre-Nord & Kiénna Yarcé & $1,000 \mathrm{~g}$ \\
\hline $\mathrm{CO} 2$ & Soudano-Sahélienne & Centre-Ouest & Mouna2 & $1,000 \mathrm{~g}$ \\
\hline S3 & Sahélienne & Sahel & Pougouzaibaogo & $1,000 \mathrm{~g}$ \\
\hline $\mathrm{CO} 1$ & Soudano-Sahélienne & Centre-Ouest & Mouna1 & $1,000 \mathrm{~g}$ \\
\hline E3 & Soudano-Sahélienne & Est & Kouaré & $0,000 \mathrm{~g}$ \\
\hline $\mathrm{E} 2$ & Soudano-Sahélienne & Est & Nadiagou & $0,000 \mathrm{~g}$ \\
\hline E1 & Soudanienne & Est & Kompienga & $0,000 \mathrm{~g}$ \\
\hline N3 & Soudano-Sahélienne & Nord & Nongdoum & $0,000 \mathrm{~g}$ \\
\hline $\mathrm{S} 1$ & Sahélienne & Sahel & Ouinpoulie & $0,000 \mathrm{~g}$ \\
\hline CE1 & Soudano-Sahélienne & Centre-Est & Gomtenga & $0,000 \mathrm{~g}$ \\
\hline SO5 & Soudanienne & Sud-Ouest & Waraba-Zinkar & $0,000 \mathrm{~g}$ \\
\hline SO1 & Soudanienne & Sud-Ouest & Gorongorona & $0,000 \mathrm{~g}$ \\
\hline $\mathrm{Ca} 6$ & Soudanienne & Cascades & Kossara & $0,000 \mathrm{~g}$ \\
\hline $\mathrm{Ca} 2$ & Soudanienne & Cascades & Wolonkoto & $0,000 \mathrm{~g}$ \\
\hline \multirow[t]{3}{*}{ HB6 } & Soudanienne & Haut-bassins & Sangoulema & $0,000 \mathrm{~g}$ \\
\hline & & Moyenne & & 5,74 \\
\hline & & $\mathrm{P}$ & & $<.0001$ \\
\hline
\end{tabular}

Les valeurs suivies d'une même lettre ne sont pas significativement différentes au seuil de 5\% selon le test de Newman keuls. 
Tableau 2: Prévalence de Fusarium spp. sur le riz produit au Burkina Faso en 2018 et niveaux d'infection des semences par ces champignons.

\begin{tabular}{lcccc}
\hline Zones de collecte des échantillons & $\begin{array}{c}\text { Nombre } \\
\text { d'échantillons } \\
\text { collectés }\end{array}$ & $\begin{array}{c}\text { Nombre } \\
\text { d'échantillons } \\
\text { contaminés par } \\
\text { Fusarium spp. }\end{array}$ & $\begin{array}{c}\text { Taux de prévalence } \\
\text { de Fusarium spp. } \\
(\%)\end{array}$ & $\begin{array}{c}\text { Taux moyen } \\
\text { d'infection des } \\
\text { semences par } \\
\text { Fusarium } \text { spp. }(\%)\end{array}$ \\
\hline Zone agro-écologique Sahélienne & 5 & 4 & 80,00 & $1,40 \mathrm{~b}$ \\
\hline Zone agro-écologique Soudano-sahélienne & 36 & 32 & 88,89 & $7,72 \mathrm{a}$ \\
\hline Zone agro-écologique Soudanienne & 18 & 12 & 66,67 & $3,00 \mathrm{~b}$ \\
\hline Total & 59 & 48 & 81,35 & - \\
\hline Moyenne & - & - & - & 5,74 \\
\hline $\mathrm{p}$ (au seuil de 5\%) & - & - & - & $<0,0001$ \\
\hline
\end{tabular}

Les valeurs suivies d'une même lettre ne sont pas significativement différentes au seuil de $5 \%$ selon le test de Newman keuls.

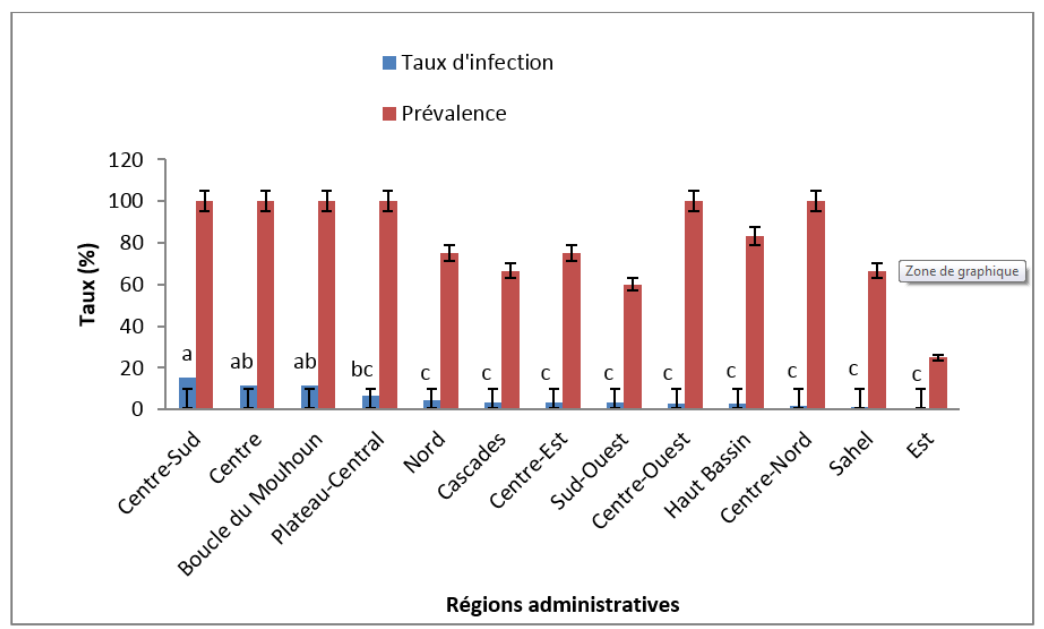

Figure 2 : Prévalence et Taux d'infection des semences de riz par Fusarium spp. dans les 13 regions administratives du Burkina Faso.

Tableau 3: Pourcentages d'échantillons de semences contaminés et taux d'infection des semences par Fusarium spp. selon la variété d'appartenance des échantillons de semences.

\begin{tabular}{lllll}
\hline Variétés de riz & $\begin{array}{l}\text { Nombre } \\
\text { d'échantillons } \\
\text { collectés }\end{array}$ & $\begin{array}{l}\text { Nombre } \\
\text { d'échantillons } \\
\text { Contaminés par } \\
\text { Fusarium } \text { spp. }\end{array}$ & $\begin{array}{l}\text { \% d'échantillons } \\
\text { contaminés par } \\
\text { Fusarium } \text { spp. }\end{array}$ & $\begin{array}{l}\text { Taux d'infection } \\
\text { des semences par } \\
\text { Fusarium spp. } \\
(\%)\end{array}$ \\
\hline FKR19 & 35 & 30 & 85,71 & $6,90 \mathrm{a}$ \\
\hline FKR45N & 3 & 1 & 33,33 & $5,00 \mathrm{a}$ \\
\hline TS2 & 15 & 13 & 86,67 & $4,90 \mathrm{a}$ \\
\hline FKR56 & 3 & 2 & 66,67 & $2,00 \mathrm{a}$ \\
\hline FKR78 & 1 & 1 & 100 & $2,00 \mathrm{a}$ \\
\hline NERICA4 & 1 & 1 & 100 & $1,00 \mathrm{a}$ \\
\hline Variété locale & 1 & 0 & 0 & $0,00 \mathrm{a}$ \\
\hline Moyenne & - & - & - & 5,74 \\
\hline p & - & - & - & 0,1495 \\
\hline
\end{tabular}

$\overline{\text { Les valeurs suivies d'une même lettre ne sont pas significativement différentes au seuil de 5\% selon le test de Newman keuls. }}$ 


\section{DISCUSSION}

Le riz est cultivé dans toutes les trois zones climatiques du Burkina Faso, à des degrés variables. Les conditions pluviométriques et édaphiques dans les zones soudaniennes et soudano-sahélienne se prêtent mieux à la culture du riz comparée à la zone sahélienne. En se référant aux statistiques agricoles disponibles (DGESS, 2019), les zones climatiques soudano-sahélienne, soudanienne et sahélienne ont respectivement fourni $65,43 \%, 31,28 \%$ et $3,28 \%$ de la production nationale de riz en 2018. Près des $2 / 3$ de la production annuelle de riz provient de la zone soudano-sahélienne. Aussi, un grand nombre d'échantillons de semences (36 échantillons) a pu être collecté dans cette zone, étant donné la disponibilité des semences auprès des producteurs.

La majeure partie de la zone sahélienne étant peu favorable à l'agriculture, la production de riz est très faible au regard des conditions climatiques drastiques (sècheresse, pauvreté des sols, érosion, manque de périmètres irrigués, attaques acridiennes). Les études ont montré que les sols de la zone sahélienne sont pauvres en matières organiques (Kissou et al., 2018). Cette situation pourrait expliquer le nombre relativement faible d'échantillons collectés dans cette partie du pays (5 échantillons). Quant à la zone soudanienne, relativement plus humide, des cultures comme le coton, le maïs et l'igname sont prioritaires au détriment du riz, ce qui pourrait également justitifier le nombre relativement peu élevé d'échantillons (18 échantillons) collectés dans cette partie du territoire. Loin d'être une collecte exhaustive d'échantillons de semences, cet échantillonnage peut néanmoins être considéré comme représentatif du territoire du fait que les échantillons proviennent des trois zones agro écologiques du pays.

Une ou plusieurs espèces appartenant au genre Fusarium ont été recensées dans 48 des 59 échantillons de semences de riz collectés. Ainsi, des grains apparemment sains, choisis comme semences par les producteurs, hébergent en leur sein une ou plusieurs espèces de Fusarium. Une forte proportion des échantillons de semences collectés à travers le pays $(81,3 \%)$ a été contaminée par ce groupe de champignons. Les champignons du genre
Fusarium sont connus responsables de pourritures de semences, de rabougrissements de plantules, de chlorose et d'élongation foliaire sur le riz (Jeon et al., 2013). Ce groupe de champignons a été fréquemment rencontré dans toutes les trois zones agroécologiques (66,67-88,89\% des échantillons collectés étaient contaminés) et dans toutes les 13 régions administratives (25-100\% des échantillons étaient infectés), indiquant le caractère cosmopolite de ces champignons. Les champignons ont été répertoriés dans les semences à des taux allant de 1 à $41 \%$. Selon Karim et al. (2016), les facteurs géographiques, y compris le climat, sont d'une importance capitale pour la diversité des espèces de Fusarium. Des observations similaires ont été faites en Asie sur des semences de riz avec des incidences allant de $3 \%$ à $80 \%$ (Jeon et al., 2013).

Parmi les trois zones étudiées, la zone Soudano-Sahélienne a enregistré la plus forte prévalence. Cela pourrait être dû au fait que cette zone occupe la plus grande partie du territoire, et a, par conséquent, fourni le plus grand nombre d'échantillons de riz collectés. En effet, les régions du Centre, la Boucle du Mouhoun, du Plateau central, du Centre-ouest, du Centre-nord avec 100\% d'échantillons de semences attaqués ainsi que le Centre-est (75\%) et l'Est (25\%) qui sont toutes situées dans la zone soudano-sahélienne ont influencé la prévalence moyenne de Fusarium spp. de cette zone. En outre, les conditions climatiques particulières de cette zone, avec une pluviométrie annuelle comprise entre 600 et $900 \mathrm{~mm}$, semblent favorables à l'expansion des champignons. Les sols des dépressions sont très pauvres en argile (moins de $2 \%$ sur l'ensemble des horizons) alors que ceux des bas-fonds et des longs glacis en sont très riches (en moyenne 15\%) (Dapola et al, 2008). Boudoudou et al. (2009) ont montré que les sols argileux à forte capacité d'échange cationique et riche en éléments nutritifs favorisent l'installation et le développement des Fusarium.

La zone Sahélienne comportant les régions du Sahel et du Nord, a une prévalence inferieure à la zone soudanienne constituée des régions du Centre-sud, des Hauts-bassins, des Cascades et du Sud-ouest. Dossa et al. (2019), confirment que les facteurs climatiques 
agissent sur le développement des Fusarium. Egalement certains éléments minéraux du sol agissent aussi sur le développement des Fusarium en inhibant la croissance mycélienne et la production des conidies (Attrassi et Rahouti, 2016).

Au total, six (6) variétés améliorées de riz et une variété locale sont utilisées par les producteurs dans les zones de production concernées par l'étude, les variétés les plus cultivées étant FKR 19 avec 35 échantillons et TS2 avec 15 échantillons collectés. Pour chacune de ces deux variétés, le nombre d'échantillons contaminés a été relativement élevé (30 échantillons pour FKR19 et 13 pour TS2). Bien que les analyses statistiques n'aient révélé aucune différence significative entre les niveaux d'infection des semences de ces variétés, les semences de la variété FKR19 avec en moyenne $6,90 \%$ de taux d'infection ont eu tendance à être plus attaquées par Fusarium spp. par rapport aux semences des autres variétés $(0-5 \%$ de taux d'infection). Il est à noter que la seule variété locale collectée a été rencontrée dans la zone soudano-sahélienne, dans la région du Centre-est (localité de Gomtenga) et a été indemne d'infection.

Dans la présente étude, les semences collectées dans la zone sahélienne ont été faiblement infectées ( $0-3 \%)$ comparées à celles collectées dans la zone soudanienne (0-12\%) et soudano-sahélienne (0-41\%). Le climat, beaucoup plus sec dans la zone sahélienne que dans les zones soudano-sahélienne et soudanienne, pourrait justifier la faible représentation des champignons dans la zone sahélienne. Selon La présence relativement importante de Fusarium spp. dans les semences originaires de la zone soudano-sahélienne pourrait également être due au fait que les évaluations ont porté sur un nombre important d'échantillons de semences provenant de cette zone agroécologique. Répandus dans la plupart des zones de culture du riz, les champignons du genre Fusarium ont été retrouvés associés aux semences de riz en Inde, Indonésie, Népal, Pakistan, Bangladesh, Egypte, Ghana, Nigeria, Bénin, à des taux élevés (Kini et al., 2002; Mathur et Manandhar, 2003). Certaines espèces de Fusarium spp. appartenant au complexe Gibberella fujikuroi ont été associées à la maladie de Bakanae ou gigantisme du riz (Sachin et al., 2019). La présence de ces champignons dans les grains de riz, en quantité relativement importante, est alarmante d'autant plus que le genre Fusarium est également reconnu produire des mycotoxines dangereuses pour la santé (Desjardins et al., 2000 ; Mathur et Manandhar, 2003 ; Latiffah et al., 2013). La présente étude donne des informations sur la présence de Fusarium spp. associé aux semences de riz produites au Burkina Faso, confirmant ainsi les résultats antérieurement rapportés par Kini et al., 2002 et Mathur et Manandhar, 2003. Une identification formelle des espèces de Fusarium associées aux semences collectées ainsi que leur caractérisation pathogénique permettraient une meilleure connaissance des principales espèces inféodées au riz au Burkina Faso et les maladies qu'elles provoquent. Ces informations sont indispensables pour la mise en place de stratégies adéquates de gestion de ces pathologies. Le gigantisme étant l'une des maladies importantes du riz causées par un complexe d'espèces de Fusarium, des précautions devraient être prises pour éviter l'expansion de cette pathologie dans les zones de production du riz au Burkina Faso. Les champignons du genre Fusarium étant transmissibles par les semences, les informations obtenues permettraient également de prendre des mesures phytosanitaires pour lutter contre les espèces pathogènes et circonscrire les foyers d'infection afin d'éviter leur dissémination et leur expansion dans d'autres zones de production de riz à travers les échanges de semences.

\section{Conclusion}

L'étude a montré que $81,35 \%$ des échantillons de semences de riz collectés au Burkina Faso ont été contaminés par Fusarium spp., chaque échantillon contenant en moyenne $5,34 \%$ de semences infectées. Elle fournit également des indications sur la qualité sanitaire des semences de riz produites dans le pays pendant la saison 2017-2018. Il est important de mettre en place un système de gestion phytosanitaire pour réduire cette prévalence des agents pathogènes afin de permettre aux riziculteurs de produire des semences de qualité et de prévenir une éventuelle épidémie de maladies telle que le gigantisme du riz. Les travaux futurs consisteront à identifier sur le plan moléculaire 
les espèces de Fusarium spp. associées aux semences de riz au Burkina Faso et à évaluer leur pathogénicité en vue de déterminer celles impliquées dans le gigantisme du riz. Au regard de l'importance de la filière riz pour le pays, des stratégies de lutte intégrée doivent être envisagées pour sécuriser la production aussi bien en quantité et qu'en qualité.

\section{CONFLIT D'INTERETS}

Les auteurs déclarent qu'il n'y a aucun conflit d'intérêts.

\section{CONTRIBUTIONS DES AUTEURS}

NWK est l'investigateur principal du projet de recherche et a rédigé le manuscrit. ZEP a été notre co-encadreur scientifique et a corrigé le manuscrit. TIG, NWL et KK ont aidé à la correction du manuscrit. SM a été l'encadreur scientifique au niveau de l'école doctorale Sciences et Technologies de l'Université Joseph KI-ZERBO.

\section{RÉMERCIEMENTS}

Les auteurs adressent leurs sincères remerciements au Projet Riz Pluvial (PRP) du Ministère de l'Agriculture et des Aménagements Hydro-Agricoles du Burkina Faso et le personnel du laboratoire de Phytopathologie de l'Institut de l'Environnement et de Recherche Agricole (INERA) pour leur contribution à la réalisation de cette étude.

\section{REFERENCES}

Attrassi K, Rahouti M. 2016. Effet de composés calciques inorganiques sur le développement in vitro de moisissures isolées d'agrumes après la récolte. Bulletin de la Société Royale des Sciences de Liège, 8: 263-275.

Badolo F, Traore F. 2015. Impact of rising world rice prices on poverty and inequality in Burkina Faso. Development Policy Review., 33 (2): 221-244. DOI: 10.1111/dpr.12099.

Bashyal BM, Aggarwal R, Sharmal S, Gupta S, Singh UB. 2016. Single and combined effects of three Fusarium species associated with rice seeds on the severity of Bakanae disease of rice. Journal of Plant Pathology, 98 (3): 405-412.
Bila NK. 2015. Revue Documentaire des Etudes sur l'état des Lieux de la Filière riz au Burkina Faso. Comité Inter Professionnel du Riz (CIR-B), Burkina Faso, 80p.

Boudoudou H, Hassikou R, Ouazzani Touhami A, Badoc A, Douria A. 2009. Paramètres physicochimiques et flore fongique des sols de rizières marocaines. Bull. Soc. Pharm. Bordeaux, 148: 17-44.

CIR-B. 2011. Stratégie de mobilisation de ressources: Analyse de la filière riz au Burkina Faso. Comité Inter Professionnel du Riz (CIR-B), Burkina Faso, 72p.

Dapola EC, Yacouba H, Yonkeu S. 2008. Unités morphopédologiques et gestion de la fertilité des sols dans le centre-nord du Burkina Faso par les populations locales. Int. J. Biol. Chem. Sci., 2(3): 306-315.

Desjardins AE, Manandhar HK, Plattner RD, Manandhar GG, Poling SM, Maragos CM. 2000. Fusarium species from Nepalese rice and production of mycotoxins and gibberellic acid by selected species. Appl Environ Microbiol., 66 (3): 1020-1025. DOI: 10.1128/AEM.66 (3) 1020-1025.2000.

DGESS. 2019. Résultats définitifs de production de la campagne agricole 2018/2019. Direction Générale des Etudes et des Statistiques Sectorielles. Ministère de l'agriculture et des aménagements hydro-agricoles, Burkina Faso, 16p.

Dossa JSB, Togbe EC, Pernaci M, Agbossou EK, Ahohuendo BC. 2019. Effet des facteurs de l'environnement sur les Fusarium pathogènes des plantes cultivées. Int. J. Biol. Chem. Sci., 13(1): 493-502. DOI: 10.4314/ijbcs.v13i1.39.

Gupta AK, Solanki IS, Bashyal Y, Singh Y, Srivastara K. 2015. Bakanae of rice: an emerging disease in Asia. The Journal of Animal \& Plant Sciences., 25(6): 14991514.

Jeon Y-A, Yu SH, Lee YY, Park HJ, Lee S, Sung JS, Kim YG, Lee HS. 2013. Incidence, Molecular Characteristics and Pathogenicity of Gibberella fujikuroi Species Complex Associated with Rice Seeds from Asian Countries. Mycobiology., 41(4): 225-233. DOI: 10.5941/MYCO.2013.41.4.225. 
Karim NFA, Mohd M, Nor NMIM, Zakaria L. 2016. Saprophytic and potentially pathogenic Fusarium species from peat soil in Perak and Pahang. Trop Life Sci Res., 27: 1-20.

Kini KR, Leth V, Mathur SB. 2002. Genetic variation in Fusarium moniliforme isolated from seeds of different host species from Burkina Faso based on random amplified polymorphic DNA analysis. Journal of Phytopathology., 150 (4/5): 209-212. DOI: 10.1046/j.14390434.2002.00739.x

Latiffah Z, Nurul huda MS, Tengu ahmed akram TMA. 2013. Characterization of Fusarium semitectum isolates from vegetable fruits. Sains Malaysiana., 42(5): 629-633.

Ma LJ, Geiser DM, Proctor RH, Rooney AP, O'donnell K, Trail F, Gardiner DM, Mannersn JM, Kazan K. 2013. Fusarium Pathogenomics. Annual Review of Microbiology., 67: 399-416. DOI: 10.1146/annurev-micro-092412-155650.

Kissou R, Gnankambary Z, Nacro HB, Sedogo MP. 2018. Classification locale et utilisation des sols en zone sahélienne au Burkina Faso. Int. J. Biol. Chem. Sci., 12(1): 610-617. DOI: 10.4314/ijbcs.v12i1.46.

MASA. 2013. Situation de la filière riz au Burkina Faso. Ministère de l'Agriculture et de la Sécurité alimentaire (MASA), Burkina Faso, 12p.

Mathur SB, Kongsdal O. 2003. Common laboratory seed health testing methods for detecting fungi. First edition; Danish Government Institute of Seed Pathology for Developing Countries, Copenhagen, Denmark, 425p.

Mathur SB, Manandhar HK. 2003. Fungi in Seeds Recorded at the Danish Government Institute of Seed Pathology for Developing Countries ( $1^{\text {st }}$ Edn). Danish Government Institute of Seed Pathology for Developing Countries: Copenhagen, Denmark, 814 p.
MECV. 2007. Programme d'action national d'adaptation à la variabilité et aux changements climatiques (PANA du Burkina Faso). Secrétariat Permanent du Conseil National pour l'environnement et le Développement Durable. Ministère de l'Environnement et du Cadre de Vie (MECV), Burkina Faso, 96p.

Ouedraogo I, Wonni I, Sérémé D, Kaboré KB, 2016. Survey of Fungal Seed-Borne Diseases of Rice in Burkina Faso. International Journal of Agriculture Innovations and Research, 5(3): 476-480.

Ouedraogo M, Dakouo D. 2017. Evaluation de l'adoption des variétés de riz NERICA dans l'Ouest du Burkina Faso. African Journal of Agricultural and Resource Economics, 12 (1): 1-16.

Raghu S, Manoj KY, Prabhukarthikeyan SR, Mathew SB, Srikanta L, Mayabini J. 2018. Occurance, pathogenicity, characterization of Fusarium fujikuroi causing rice bakanae disease from Odisha and in vitro management. Oryza., 55 (1): 214-223. DOI: $\quad 10.5958 / 2249$ 5266.2018.00025.5.

Sachin KJ, Kamal K, Mukesh D, Sanchita P. 2019. Occurrence of Bakanae Disease of Rice in Western Uttar Pradesh, India. Int. J. Curr. Microbiol. App. Sci., 8(5): 207212.

DOI: 10.20546/ijcmas.2019.805.025.

Seck PA, Toure AA, Coulibaly JY, Diagne A, Wopereis MCS. 2013. Impact of rice research on income, poverty and food security in Africa: an ex-ante analysis. Realizing Africa's Rice Promise. CAB International, Wallingford, R-U: 24-33.

Van oort PAJ, Saito K, Tanaka A, AmovinAssagba E, Van Bussel LGJ, Vanwart J, Degroot H, Vanlttersum MK, Cassman KG, Wopereis MCS. 2015. Assessment of rice self-sufficiency in 2025 in eight African countries. Global Food Security, 5(2015): $\quad 39-49$. DOI: 10.1016/j.gfs.2015.01.002. 